\title{
Aplicação de produtos biológicos estimulantes no desenvolvimento vegetal em
}

\section{hortaliças}

\author{
Application of biological products stimulating plant development in vegetables \\ Aplicación de productos biológicos estimulantes del desarrollo vegetal en hortalizas
}

Recebido: 08/09/2021 | Revisado: 14/09/2021 | Aceito: 15/09/2021 | Publicado: 17/09/2021

\author{
Joaquim Souto Silva Júnior \\ ORCID: https://orcid.org/0000-0003-2404-5126 \\ Universidade Estadual Paulista, Brasil \\ E-mail: joaquim.souto@unesp.br \\ Marco Eustáquio de Sá \\ ORCID: https://orcid.org/0000-0002-0588-0356 \\ Universidade Estadual Paulista, Brasil \\ E-mail: marco.sa@unesp.br
}

\begin{abstract}
Resumo
O uso de produtos estimulantes na produção de olerícolas é de grande importância para um melhor estabelecimento de plântulas no campo, fator diretamente relacionado com a germinação das sementes. O objetivo deste estudo consistiu em verificar os efeitos de diferentes produtos biológicos estimulantes do desenvolvimento vegetal, como Ascophylum nodosum, Ecklonia maxima, Brutal ${ }^{\circledR}$ (Complexo de microorganismos) e Serenade® (Bacillus subtilis) no desempenho de hortaliças, em comparação com a embebição das sementes com Água e Nitrato de Potássio (KNO3 $0,2 \%$ ). Os produtos foram aplicados às sementes na dose de $150 \mathrm{~mL} / \mathrm{kg}$ de sementes, fazendo-se a mistura manualmente até a uniformização para posterior semeadura. Foram utilizadas sementes de quatro cultivares de alface, Americana, Aurélia, Crespa Repolhuda e Crespa de Verão e seis tratamentos formando um fatorial 6x4 em um delineamento inteiramente casualizado. Foram realizados os testes de germinação, primeira contagem, teste de frio, teste de envelhecimento e massa de matéria seca de plântulas. O Teste de frio evidenciou melhor desempenho das sementes em relação ao teste de envelhecimento acelerado e matéria seca de plântulas. Nas sementes de alto vigor não ficaram evidentes os efeitos dos tratamentos das sementes sobre o desempenho. Concluiu-se que os produtos influenciaram na primeira contagem de germinação e germinação total, índice de velocidade de germinação com melhoria de desempenho nas sementes menos vigorosas.
\end{abstract}

Palavras-chave: Horticultura; Alface; Biorreguladores; Tratamento de sementes.

\begin{abstract}
The use of stimulating products in the production of vegetables is of great importance for a better establishment of seedlings in the field, a factor directly related to seed germination. The aim of this study was to verify the effects of different biological products stimulating plant development such as Ascophylum nodosum, Ecklonia maxima, Brutal ${ }^{\circledR}$ (Complex of microorganisms) and Serenade ${ }^{\circledR}$ (Bacillus subtilis) on the performance of vegetables, in comparison with the imbibition of seeds with Water and Potassium Nitrate $(0.2 \% \mathrm{KNO} 3)$. The products were applied to the seeds at a dose of $150 \mathrm{~mL} / \mathrm{kg}$ of seeds, making the mixture manually until uniform for later sowing. Seeds of four cultivars of lettuce, Americana, Aurélia, Crespa Repolhuda and Crespa de Verão were used and six treatments forming a 6x4 factorial in a completely randomized design. Germination tests, first count, cold test, accelerated test and seedling dry matter were carried out. It was concluded that the products influenced the first germination count and total germination, germination speed index with improved performance in less vigorous seeds. The cold test showed better seed performance in relation to the accelerated test and seedling dry matter test. In high vigor seeds, the effects of seed treatments on performance were not evident.
\end{abstract}

Keywords: Horticulture; Lettuce; Bioregulators; Seed treatment.

\section{Resumen}

El uso de productos estimulantes en la producción de hortalizas es de gran importancia para un mejor establecimiento de las plántulas en el campo, factor directamente relacionado con la germinación de las semillas. El objetivo de este estudio fue verificar los efectos de diferentes productos biológicos estimulantes del desarrollo vegetal como Ascophylum nodosum, Ecklonia maxima, Brutal® (Complejo de microorganismos) y Serenade® (Bacillus subtilis) sobre el desempeño de vegetales, en comparación con la imbibición. de semillas con Agua y Nitrato de Potasio (0.2\% KNO3). Los productos se aplicaron a las semillas a una dosis de $150 \mathrm{~mL} / \mathrm{kg}$ de semillas, haciendo la mezcla manualmente hasta uniformidad para su posterior siembra. Se utilizaron semillas de cuatro cultivares de lechuga, Americana, Aurélia, Crespa Repolhuda y Crespa de Verão y seis tratamientos formando un factorial 6x4 en un diseño 
completamente al azar. Se realizaron pruebas de germinación, primer conteo, prueba en frío, prueba de envejecimiento y materia seca de la plántula. Se concluyó que los productos influyeron en el primer conteo de germinación y en la germinación total, índice de velocidad de germinación con mejor desempeño en semillas menos vigorosas. La prueba en frío mostró un mejor rendimiento de la semilla en relación con la prueba de envejecimiento acelerado y materia seca de la plántula. En semillas de alto vigor, los efectos de los tratamientos de semillas sobre el rendimiento no fueron evidentes.

Palabras clave: Horticultura; Lechuga; Biorreguladores; Tratamiento de semillas.

\section{Introduçãa}

Várias plantas hortícolas possuem um ciclo relativamente rápido, com isso, há uma alta movimentação de caixa, o que é favorável ao produtor, gerando alta lucratividade por hectare cultivado e por hectare/ano (Vilela \& Luengo, 2017). A alface (Lactuca sativa L.) é a hortaliça folhosa mais consumida no mundo (Costa \& Sala, 2005), apresenta grande valor nutricional, alto teor de fibras, baixo valor calórico e fácil digestão, sendo amplamente utilizada em dietas, por disponibilizar vitaminas e sais minerais (Mota et al., 2012); além disso, estudos comprovam que a alface é uma hortaliça que apresenta funções tranquilizantes (Fonseca et al., 2013). O cultivo da alface do grupo crespa é predominante no Brasil, sendo 70\% do mercado, já a americana corresponde a $15 \%$, a lisa $10 \%$ e as outras (vermelha, mimosa e romana) somam um total de $5 \%$ do mercado (Costa \& Sala, 2005).

De acordo com dados fornecidos pela Associação Brasileira do Comércio de Sementes e Mudas (ABCSEM), a cultura da alface é responsável por movimentar em média, um total de 8 bilhões de reais com uma produção de mais de 1,5 milhão de toneladas por ano (ABCSEM, 2013). Segundo o censo Agropecuário do Instituto Brasileiro de Geografia e Estatística (IBGE), os estabelecimentos produtores de alface no Brasil somam um total de 108.603 unidades, e que produzem um total de 908.186 toneladas por ano (IBGE, 2017).

A cultivar americana vem tendo uma grande importância, resultando em uma crescente no mercado brasileiro. Geralmente cultivada em pequenas propriedades de agricultura familiar, de forma intensiva, comumente localizadas em áreas periurbanas ou nos cinturões verdes dos grandes centros urbanos (Costa \& Sala, 2005). A cultivar americana é uma planta herbácea, com caule reduzido, sem ramificação, onde se prendem as folhas. As folhas mais velhas têm a coloração verdeescuro, já as mais novas podem variar entre amarela ou branca, são crocantes e interligadas de forma semelhante ao repolho. Quando comparada a outras cultivares de alface, a americana apresenta maior vida útil pós-colheita, possibilitando o transporte a longas distâncias (Decoteau et al., 1995).

As cultivares de alfaces do tipo crespa, como a Alface Crespa de Verão e também a Crespa Repolhuda, são preferencialmente usadas pelos produtores que residem em ambientes mais quentes, por serem consideradas adaptadas ao cultivo no verão. Essas cultivares geralmente tem maior resistência a doenças e ao pendoamento precoce, e também apresentam boa disposição das folhas, resistência ao transporte, e um período pós-colheita mais longo, além de melhor palatabilidade (Rodrigues et al., 2007). As condições do ambiente têm enorme influência no comportamento da alface; temperaturas acima de $20^{\circ} \mathrm{C}$ podem levar ao pendoamento precoce da alface, o qual pode ser agravado com o aumento da temperatura, promovendo variação de comportamento entre cultivares (Viggiano, 1990).

Ainda que seja uma cultura cultivada em uma enorme gama de regiões brasileiras, existem algumas restrições quanto ao seu cultivo, devido a sua sensibilidade para condições adversas, como temperatura, umidade e precipitação (Gomes et al., 2005). Em circunstâncias meteorológicas desfavoráveis, como baixas temperaturas $\left(<10^{\circ} \mathrm{C}\right)$ e chuvas constantes, a planta tem seu crescimento retardado e ainda podem danificar as plantas. Já temperaturas elevadas $\left(>20{ }^{\circ} \mathrm{C}\right)$, com alta radiação solar, podem ocorrer pendoamentos precoce das plantas, além de poder provocar queima da bordadura das folhas externas, também pode acarretar em cabeças pouco compactas e contribuir para uma deficiência de cálcio denominada "Tipburn" (Turini et al., 2011). 
Os inúmeros trabalhos com o gênero Bacillus, vem dando grande importância à área de controle biológico de doenças, onde o foco das avaliações são averiguar diversas espécies deste gênero, assim como o B. subtilis (Araujo, 2008). Essas espécies estudadas de Bacillus sp., como rizobactérias, tem o intuito de promover crescimento de plantas e vem apresentando enorme importância nos últimos anos, sendo utilizados em uma alta gama de estudos, todavia em alguns trabalhos tem sido utilizado algumas espécies de bactérias isoladas que não foram selecionadas para tal atividade em culturas agrícolas especificas (Araujo, 2008).

Com isso, o objetivo deste estudo consistiu em verificar os efeitos de diferentes produtos biológicos estimulantes do desenvolvimento vegetal no desempenho de hortaliças, em comparação com a embebição das sementes com água e KNO3 $0,2 \%$.

\section{Metodologia}

O experimento foi conduzido em laboratório, pertencente ao Campus Central da UNESP, localizada no Município de Ilha Solteira/SP, com coordenadas de $20^{\circ} 25^{\prime} 30,86^{\prime \prime}$ 'S e $51^{\circ} 21^{\prime} 20,23^{\prime \prime}$ W, e altitude de $347 \mathrm{~m}$.

O delineamento experimental utilizado foi o inteiramente casualizado (DIC) em esquema fatorial $4 \times 6$, foram utilizadas quatro cultivares de alface (Lactuca sativa L.): Crespa Repolhuda, Crespa de Verão, Americana e Aurélia. Além disso, foram utilizadas seis substâncias no tratamento das sementes: Água (testemunha), Nitrato de Potássio (KNO3 0,2\%), Ascophylum nodosum, Ecklonia maxima, Brutal ${ }^{\circledR}$ (Complexo de microorganismos) e Serenade $($ Bacillus subtilis), com 4 repetições.

Na composição de cada tratamento foram utilizados $1,5 \mathrm{~mL}$ de produto misturado em $4,5 \mathrm{~mL}$ de água, onde em seguida, com o auxílio de uma pipeta de precisão, aplicou-se $1,5 \mathrm{~mL}$ da solução em $1 \mathrm{~g}$ de sementes, as quais foram acondicionadas em sacos plásticos de modo que, após a aplicação dos produtos ficassem uniformemente tratadas.

Nitrato de Potássio - a solução de KNO3 0,2\% é recomendada pelas regras para análise de sementes para quebra de dormência (BRASIL, 2009) e, portanto, como fator para promover a germinação.

Ascophylum nodosum (35\%) - fertilizante líquido, totalmente solúvel em água, produzido com alta concentração de algas, como fonte natural de citocininas, giberelinas e auxinas, as quais possuem a capacidade de promover a divisão e expansão celular.

Ecklonia maxima - fertilizante líquido totalmente solúvel em água com a presença das algas Ecklonia maxima que possui alta taxa de fito-hormônios, dentre elas a auxina.

Brutal ${ }^{\circledR}$ (Complexo de microorganismos) - fertilizante orgânico composto classe A líquido, que pode ser usado em todas as culturas, além de possuir certificado para o uso em agricultura orgânica.

Serenade ${ }^{\circledR}$ (Bacillus subtilis) - é um fungicida bactericida microbiológico que possui múltiplos modos de ação. Os lipopeptídeos produzidos pelo Bacillus subtilis QST713 presentes na formulação, atuam na membrana celular das estruturas reprodutivas do fungo, provocando sua deformação e produzindo rupturas. O Bacillus subtilis também age por competição de espaço e nutrientes na superfície vegetal da planta e no solo junto ao sistema radicular.

As sementes das cultivares (Crespa Repolhuda, Crespa de Verão, Americana e Aurélia) foram submetidas ao teste de germinação e ao teste de frio, onde foram semeadas sobre papel com quatro repetições de 50 sementes para cada tratamento. As caixas foram acondicionadas em câmara de germinação com temperatura controlada à $25^{\circ} \mathrm{C}$ para o teste de germinação, já para o teste de frio os "gerbox" foram acondicionados a uma temperatura de $10^{\circ} \mathrm{C}$ por 7 dias e posteriormente levada para germinador a $25^{\circ} \mathrm{C}$.

A primeira contagem de germinação foi realizada ao terceiro dia, e as contagens subsequentes foram também realizadas a cada 3 dias até a estabilização do mesmo, quando então foi calculada a porcentagem de germinação total. 
Foi mensurado o índice de velocidade de germinação (IVG), calculando segundo a fórmula proposta por Maguire (1962) onde, $\mathrm{IVG}=\mathrm{E} 1 / \mathrm{N} 1+\mathrm{E} 2 / \mathrm{N} 2+\ldots+\mathrm{En} / \mathrm{Nn}\left(\mathrm{En}=\mathrm{n}^{\circ}\right.$ de sementes germinadas no dia e $\mathrm{Nn}=\mathrm{n}^{\circ}$ de dias da semeadura $)$.

Para o teste de envelhecimento, as sementes devidamente tratadas foram colocadas em "gerbox" com tela sob solução salina saturadas de $\mathrm{NaCl}$ (40g de $\mathrm{NaCl}$ em $100 \mathrm{~mL}$ de água destilada) e colocadas em uma câmara B.O.D. com temperatura à $41^{\circ} \mathrm{C}$ por $72 \mathrm{~h}$ (TEKRONY, 1995), posteriormente foram submetidas ao teste de germinação com as sementes envelhecidas, com contagem de germinação no quarto dia após a semeadura.

Nas amostras coletadas na primeira contagem de cada teste, foram selecionadas aleatoriamente 10 plântulas; as mesmas foram acondicionadas em sacos de papéis devidamente identificados, para serem colocadas em estufa de ventilação forçada a uma temperatura de $65^{\circ} \mathrm{C}$ até atingir massa constante. Em seguida as amostras foram pesadas em balança de precisão $0,001 \mathrm{~g}$, sendo os dados utilizados para determinar massa seca total.

Os dados foram submetidos à análise de variância, utilizando o programa estatístico SISVAR, realizando-se o teste de Tukey entre cultivares e produtos e realizando o desdobramento quando necessário. Utilizou-se a seguinte tabela de análise de variância (Tabela 1).

Tabela 1. Análise de variância.

\begin{tabular}{cc} 
Causas de variação & g.l. \\
\hline Cultivares & 3 \\
Produtos & 5 \\
Cultivares x Produtos & 15 \\
Resíduo & 72 \\
\hline Total & 95 \\
\multicolumn{2}{c}{ Fonte: Autores. }
\end{tabular}

\section{Resultados e Discussão}

A germinação das sementes da cultivar Americana foi estatisticamente superior à germinação das demais cultivares de alface (Tabela 2), isso pode ter se dado devido à qualidade do lote, contudo observou-se que para a cultivar Aurélia, os tratamentos com $\mathrm{KNO} 3$ e com o produto Brutal ${ }^{\circledR}$ apresentaram incrementos consideráveis em sua primeira contagem de germinação. $\mathrm{O}$ uso de apenas água apresentou menor número de plantas germinadas nas cultivares Aurélia, Crespa Repolhuda e também para a Crespa de Verão, na primeira contagem indicando a possibilidade de dormência nas sementes destas cultivares.

Uma pequena taxa de germinação na primeira contagem é um fator inadequado quando se pensa na uniformidade das mudas, uma vez que isto irá gerar um desenvolvimento desigual, afetando tanto a produção como a comercialização do material. 
Tabela 2. Primeira contagem de germinação de cultivares de alface em função da aplicação de produtos biológicos estimulantes de desenvolvimento vegetal, UNESP Ilha Solteira (SP), 2021.

\begin{tabular}{|c|c|c|c|c|c|c|}
\hline \multirow{2}{*}{ Cultivares } & \multicolumn{6}{|c|}{ Tratamentos } \\
\hline & T1 & $\mathbf{T} 2$ & $\mathbf{T 3}$ & $\mathbf{T 4}$ & T5 & T6 \\
\hline $\mathrm{AA}$ & $6,00 \mathrm{Dc}$ & $45,50 \mathrm{Aab}$ & $4,50 \mathrm{Dd}$ & $19,75 \mathrm{Cc}$ & $44,25 \mathrm{Aa}$ & $33,25 \mathrm{Bb}$ \\
\hline ACR & $4,50 \mathrm{Bc}$ & $14,50 \mathrm{Ac}$ & $15,25 \mathrm{Ac}$ & $12,00 \mathrm{Abc}$ & $11,50 \mathrm{Abb}$ & $11,50 \mathrm{Abc}$ \\
\hline $\mathrm{ACV}$ & $24,50 \mathrm{Cb}$ & $37,75 \mathrm{Ab}$ & $35,00 \mathrm{Abb}$ & $36,50 \mathrm{Abb}$ & $39,50 \mathrm{Aa}$ & $28,00 \mathrm{BCb}$ \\
\hline $\mathrm{AM}$ & $46,75 \mathrm{Aa}$ & $50,00 \mathrm{Aa}$ & $47,75 \mathrm{Aa}$ & $46,00 \mathrm{Aa}$ & $46,00 \mathrm{Aa}$ & $46,50 \mathrm{Aa}$ \\
\hline \multirow{3}{*}{$\mathrm{F}$} & \multicolumn{2}{|c|}{ Cultivares } & \multicolumn{4}{|c|}{$283,82^{* *}$} \\
\hline & \multicolumn{2}{|c|}{ Tratamentos $(\mathrm{T})$} & \multicolumn{4}{|c|}{$31,88^{* *}$} \\
\hline & \multicolumn{2}{|c|}{ Cultivares x T } & \multicolumn{4}{|c|}{$16,71^{* *}$} \\
\hline \multirow{2}{*}{ DMS } & \multicolumn{2}{|c|}{ Cultivares } & \multicolumn{4}{|c|}{3,30} \\
\hline & \multicolumn{2}{|c|}{ Tratamentos } & \multicolumn{4}{|c|}{8,99} \\
\hline C.V. $(\%)$ & & & \multicolumn{4}{|c|}{14,72} \\
\hline
\end{tabular}

Médias seguidas de letras diferentes maiúsculas nas linhas e minúsculas nas colunas, diferem estatisticamente entre si, Teste de Tukey, $\mathrm{p}<0.05$ ) (means followed by different uppercase letters in the lines and lowercase letters in the columns, differ statistically from each other, Tukey, p<0.05). Cultivares: ACR-Crespa Repolhuda, ACV-Crespa de Verão, AM-Americana e AA-Aurélia; Tratamentos: T1-Água (testemunha), T2-Nitrato de Potássio $\left(\mathrm{KNO}_{3}\right.$ 0,2\%), T3-Ascophylum nodosum, T4-Ecklonia 5áxima, T5-Brutal® (Complexo de microorganismos) e T6-Serenade ${ }^{\circledR}$ (Bacillus subtilis). Fonte: Autores.

Na Figura 1, observa-se que as sementes da alface Crespa de Verão e a alface Crespa Repolhuda apresentaram taxa de germinação inferior a $70 \%$ no tratamento apenas com água e que todos os outros tratamentos proporcionaram germinação superior a $80 \%$. Ao considerar a ação dos produtos biológicos, tem-se que seus benefícios na produção de hormônios como giberelinas, auxinas e citocininas podem ter propiciado um melhor desempenho das sementes, pois de acordo com Silva et al. (2016), a ação das rizobactérias proporcionam aumento na taxa de germinação, fato também constatado para os extratos de algas (Rodrigues, 2018).

As sementes das cultivares Americana e Aurélia apresentaram uma alta taxa de germinação final para todos os tratamentos, já a Alface Crespa de Verão apresentou altos índices de germinação apenas com os tratamentos com Ascophylum nodosum, Ecklonia máxima e Bacillus subtilis, o que pode ser um indicativo do efeito benéfico das bactérias diazotróficas e do extrato de algas. 
Figura 1. Média de germinação de cultivares de alface em função da aplicação de produtos biológicos estimulantes de desenvolvimento vegetal, UNESP Ilha Solteira (SP), 2021.

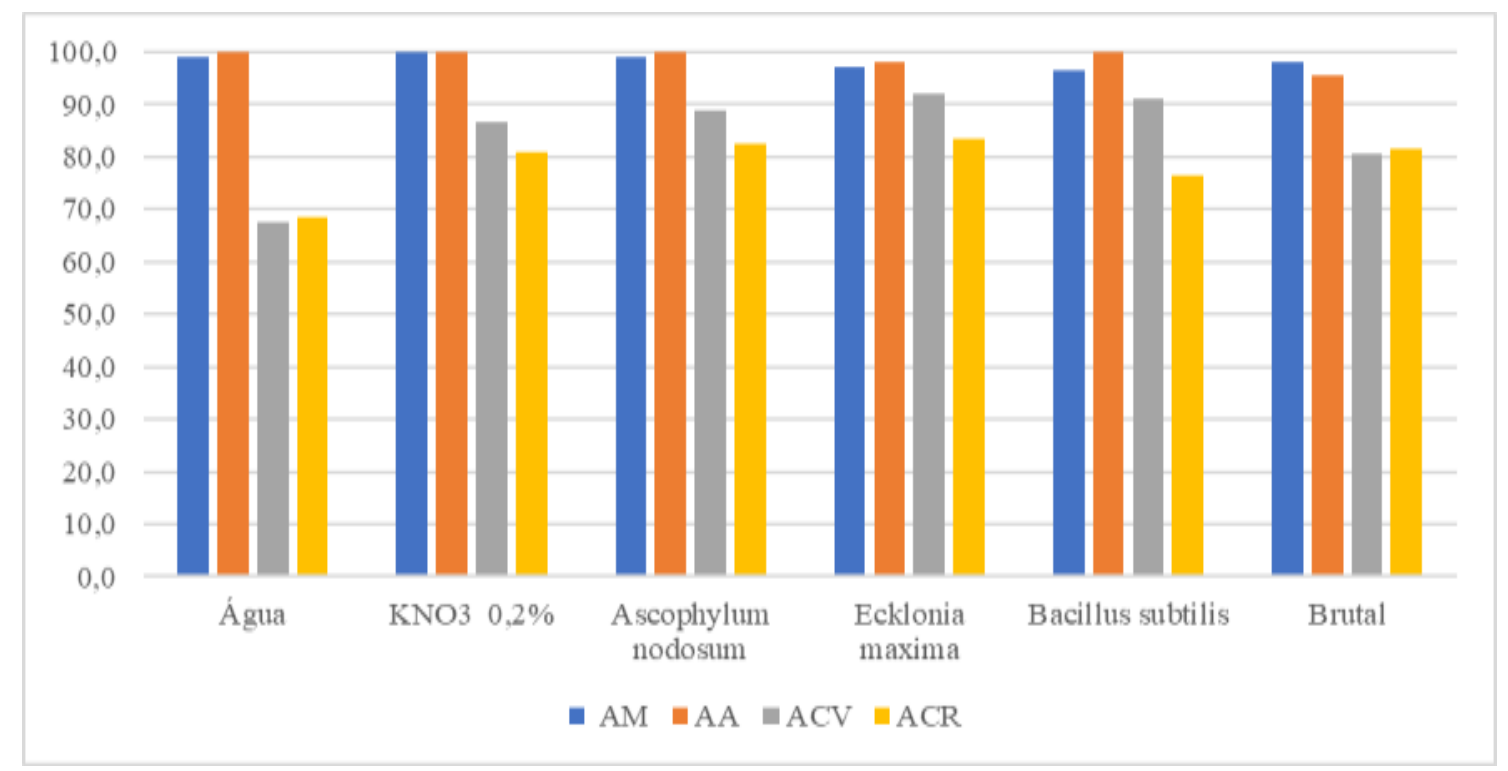

Fonte: Autores.

Analisando-se o Índice de Velocidade de Germinação, pode-se notar na Tabela 3 que a cultivar Americana se sobressaiu diante das demais cultivares em todos os tratamentos. As alfaces Crespa Repolhuda e Crespa de Verão demonstraram um IVG baixo quando tratadas apenas com água, já a Aurélia demonstrou maiores índices utilizando $\mathrm{KNO}_{3}$ e o complexo de micro-organismos da Brutal®.

No que diz respeito ao índice de velocidade de germinação, observa-se que as sementes dos dois cultivares, Alface Americana e Aurélia, que apresentaram maior desempenho na primeira contagem, também apresentaram maior índice de velocidade de germinação, confirmando o maior vigor destas sementes. Para todos os tratamentos, as sementes da Alface Americana apresentaram maior desempenho. Já as sementes da cultiva Aurélia apresentaram alto desempenho com a aplicação de $\mathrm{KNO}_{3}$ e com a Ecklonia máxima. Conforme Marcos Filho (2015), uma das manifestações do menor vigor em sementes é o maior tempo para a germinação, ou seja, uma menor velocidade germinativa.

Com exceção do observado para as sementes do cultivar de alface Americana, para todas as outras os tratamentos com as rizobactérias, $\mathrm{KNO}_{3}$ ou extrato de algas foram superiores a aplicação apenas de água, indicando que os produtos tiveram efeito no incremento da velocidade de germinação. Outro fato relevante é que nas sementes de alto vigor (Alface Americana) os tratamentos não incrementaram a germinação, indicando que a ação dos mesmos é mais relevante quando o vigor das sementes já não se encontrava em seu máximo. A ação do $\mathrm{KNO}_{3}$ 0,2\%, aumentando o índice de velocidade de germinação, pode ser um indicativo que em algumas sementes haveria necessidade da ativação da enzima citocromo-oxidase, o que poderia ser um indicativo de uma possível dormência em algumas sementes (Marcos Filho, 2015). 
Tabela 3. Índice de Velocidade de Germinação de cultivares de alface em função da aplicação de produtos biológicos estimulantes de desenvolvimento vegetal, UNESP Ilha Solteira (SP), 2021.

\begin{tabular}{|c|c|c|c|c|c|c|}
\hline \multirow{2}{*}{ Cultivares } & \multicolumn{6}{|c|}{ Tratamentos } \\
\hline & T1 & $\mathrm{T} 2$ & T3 & T4 & T5 & T6 \\
\hline AA & 6,74 Dc & $15,67 \mathrm{Aa}$ & $6,87 \mathrm{Dd}$ & $10,75 \mathrm{Cc}$ & $15,64 \mathrm{Aa}$ & $13,20 \mathrm{Bb}$ \\
\hline ACR & $5,58 \mathrm{Bc}$ & $8,63 \mathrm{Ac}$ & $9,37 \mathrm{Ac}$ & $8,79 \mathrm{Ac}$ & $8,07 \mathrm{Ab}$ & $8,66 \mathrm{Ac}$ \\
\hline $\mathrm{ACV}$ & $9,27 \mathrm{Cb}$ & $13,38 \mathrm{ABb}$ & $13,27 \mathrm{ABb}$ & $13,64 \mathrm{Ab}$ & $14,19 \mathrm{Aa}$ & $11,25 \mathrm{BCb}$ \\
\hline $\mathrm{AM}$ & $16,01 \mathrm{Aa}$ & $16,67 \mathrm{Aa}$ & $16,23 \mathrm{Aa}$ & $15,81 \mathrm{Aa}$ & $15,79 \mathrm{Aa}$ & $15,91 \mathrm{Aa}$ \\
\hline \multirow{3}{*}{$\mathrm{F}$} & \multicolumn{3}{|c|}{ Cultivares } & \multicolumn{3}{|c|}{$209,01^{* *}$} \\
\hline & \multicolumn{3}{|c|}{ Tratamentos $(\mathrm{T})$} & \multicolumn{3}{|c|}{$30,94^{* *}$} \\
\hline & \multicolumn{3}{|c|}{ Cultivares $\mathrm{x} T$} & \multicolumn{3}{|c|}{$13,68^{* *}$} \\
\hline \multirow{2}{*}{ DMS } & \multicolumn{3}{|c|}{ Cultivares } & \multicolumn{3}{|c|}{0,84} \\
\hline & \multicolumn{3}{|c|}{ Tratamentos } & \multicolumn{3}{|c|}{2,28} \\
\hline C.V. (\%) & & & & \multicolumn{3}{|c|}{9,13} \\
\hline
\end{tabular}

Médias seguidas de letras diferentes maiúsculas nas linhas e minúsculas nas colunas, diferem estatisticamente entre si, Teste de Tukey, $\mathrm{p}<0.05$ ) (means followed by different uppercase letters in the lines and lowercase letters in the columns, differ statistically from each Other, Tukey, p<0.05). Cultivares: ACR-Crespa Repolhuda, ACV-Crespa de Verão, AM-Americana e AA-Aurélia; Tratamentos: T1-Água (testemunha), T2-Nitrato de Potássio ( $\mathrm{KNO}_{3}$ 0,2\%), T3-Ascophylum nodosum, T4-Ecklonia maxima, T5Brutal ${ }^{\circledR}$ (Complexo de micro-organismos) e T6-Serenade ${ }^{\circledR}$ (Bacillus subtilis). Fonte: Autores.

Quanto à matéria seca, a cultivar Crespa Repolhuda demonstrou uma maior quantidade de matéria quando tratada apenas com água (Figura 2), o que não se repetiu quando utilizados os outros tratamentos; a Alface Aurélia apresentou baixo teor de matéria seca quando tratada com Bacillus subtilis; já a Americana teve maior volume de matéria com Ascophylum nodosum. As observações com Bacillus subtilis contrariam resultados de Zucareli et al. (2018), que relataram que a utilização de Bacillus subtilis promoveu em alface maior índice de área foliar, maior massa seca da parte aérea e contribuiu para massa seca de raiz devido a produção de fito-hormônios como auxinas e giberelinas.

Figura 2. Matéria Seca (g) de cultivares de alface em função da aplicação de produtos biológicos estimulantes de desenvolvimento vegetal, UNESP Ilha Solteira (SP), 2021.

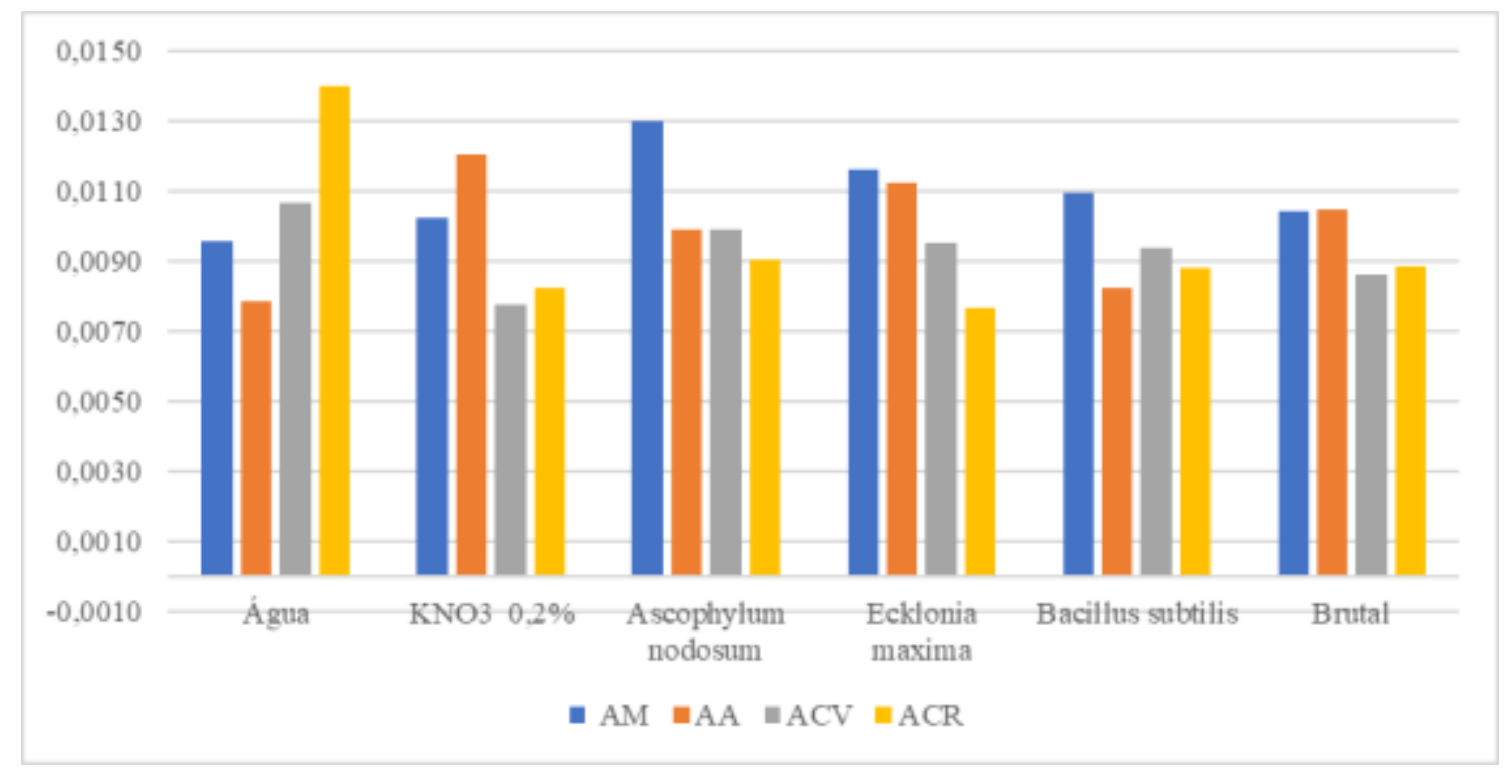

Fonte: Autores. 
A matéria seca das plântulas mostrou um comportamento com certa desuniformidade, quando se observa a primeira contagem e a velocidade de germinação. De modo geral, o vigor das sementes é avaliado por meio da utilização de vários testes, tendo-se em vista que ainda não existe um teste que seja o indicado para confirmar o vigor de determinada espécie de forma isolada e desta forma o mais adequado é a soma das informações de vários testes (Marcos Filho, 2021).

Estas variações entre cultivares e tratamentos na produção de matéria seca não deixa um indicativo para que se possa recomendar determinado tratamento e mostra também divergências do resultado deste teste em relação à germinação e primeira contagem.

Para o teste de frio, a aplicação da baixa temperatura no lote de sementes pode ter acarretado em uma melhora quanto a primeira contagem (Tabela 4), as cultivares Aurélia, Crespa de Verão e também a Americana apresentaram altos índices de germinação na primeira contagem e também não diferiram entre os tratamentos, já a Crespa Repolhuda apresentou menor número de plântulas germinadas, em especial quando tratadas com Ecklonia maxima e Serenade®. Ressalta-se que esta contagem com 3 dias visou evidenciar o vigor dos lotes uma vez que nas regras para análise de sementes (BRASIL, 2009), recomenda-se que a mesma seja realizada com 4 dias.

Os resultados de primeira contagem no teste de frio confirmaram o desempenho das sementes da alface Americana e da Aurélia, que evidenciaram um desempenho superior ao da primeira contagem de germinação para as sementes dos outros dois cultivares, indicando que a ação da baixa temperatura imposta pelo teste $\left(7\right.$ dias a $\left.10^{\circ} \mathrm{C}\right)$ favoreceu o processo germinativo das sementes com a quebra de dormência (Tabela 4). Da mesma forma, as sementes das cultivares de alface Americana e Aurélia apresentaram um alto desempenho em todos os tratamentos, próximo a 100\% de germinação, e as sementes da cultivar Alface Crespa de Verão apresentou germinação na faixa de $90 \%$. Já as sementes da cultivar alface Crespa Repolhuda apresentaram um desempenho inferior com desempenho entre $70 \%$ e $80 \%$, sendo este valor observado com o uso da água destilada indicando que os tratamentos com as rizobactérias ou com o extrato de água não promoveram incremento no desempenho das sementes.

Esta boa ação do frio é confirmada pelo índice de velocidade de germinação (Tabela 4) com desempenho semelhante ao verificado para primeira contagem do teste de frio (Tabela 4) e germinação total do teste de frio (Figura 3).

Da mesma forma que o observado para massa de matéria seca de plântulas no teste de germinação, as plântulas em teste de frio não evidenciaram grandes alterações no desempenho das sementes de forma a permitir indicar um melhor tratamento para promover o desempenho das sementes (Figura 4). 
Tabela 4. Primeira contagem de germinação no teste de frio de cultivares de alface em função da aplicação de produtos biológicos estimulantes de desenvolvimento vegetal, UNESP Ilha Solteira (SP), 2021.

\begin{tabular}{|c|c|c|c|c|c|c|}
\hline \multirow{2}{*}{ Cultivares } & \multicolumn{6}{|c|}{ Tratamentos } \\
\hline & T1 & $\mathbf{T 2}$ & T3 & T4 & T5 & T6 \\
\hline $\mathrm{AA}$ & $49,75 \mathrm{Aa}$ & $49,50 \mathrm{Aa}$ & $49,75 \mathrm{Aa}$ & $49,75 \mathrm{Aa}$ & $49,75 \mathrm{Aa}$ & $49,25 \mathrm{Aa}$ \\
\hline ACR & $31,75 \mathrm{Ab}$ & $29,75 \mathrm{ABb}$ & $27,00 \mathrm{ABCb}$ & $22,50 \mathrm{Cc}$ & $25,50 \mathrm{BCc}$ & $23,25 \mathrm{Cc}$ \\
\hline $\mathrm{ACV}$ & $45,75 \mathrm{Aa}$ & $46,25 \mathrm{Aa}$ & $45,50 \mathrm{Aa}$ & $43,25 \mathrm{Ab}$ & $43,75 \mathrm{Ab}$ & $44,00 \mathrm{Ab}$ \\
\hline $\mathrm{AM}$ & $50,00 \mathrm{Aa}$ & $49,75 \mathrm{Aa}$ & $49,75 \mathrm{Aa}$ & $50,00 \mathrm{Aa}$ & $49,25 \mathrm{Aa}$ & $50,00 \mathrm{Aa}$ \\
\hline \multirow{3}{*}{$\mathrm{F}$} & \multicolumn{3}{|c|}{ Cultivares } & \multicolumn{3}{|c|}{$491,67^{* *}$} \\
\hline & \multicolumn{3}{|c|}{ Tratamentos $(\mathrm{T})$} & \multicolumn{3}{|c|}{$3,95^{* *}$} \\
\hline & \multicolumn{3}{|c|}{ Cultivares x T } & \multicolumn{3}{|c|}{$2,05^{*}$} \\
\hline \multirow{2}{*}{ DMS } & \multicolumn{3}{|c|}{ Cultivares } & \multicolumn{3}{|c|}{1,84} \\
\hline & \multicolumn{3}{|c|}{ Tratamentos } & \multicolumn{3}{|c|}{5,02} \\
\hline \multicolumn{4}{|l|}{ C.V. $(\%)$} & \multicolumn{3}{|c|}{5,68} \\
\hline
\end{tabular}

Médias seguidas de letras diferentes maiúsculas nas linhas e minúsculas nas colunas, diferem estatisticamente entre si, Teste de Tukey, $\mathrm{p}<0.05$ ) (means followed by different uppercase letters in the lines and lowercase letters in the columns, differ statistically from each Other, Tukey, p<0.05). Cultivares: ACR-Crespa Repolhuda, ACV-Crespa de Verão, AM-Americana e AA-Aurélia; Tratamentos: T1-Água (testemunha), T2-Nitrato de Potássio ( $\mathrm{KNO}_{3}$ 0,2\%), T3-Ascophylum nodosum, T4-Ecklonia maxima, T5Brutal ${ }^{\circledR}$ (Complexo de microorganismos) e T6-Serenade ${ }^{\circledR}$ (Bacillus subtilis). Fonte: Autores.

Nota-se que todas as cultivares com exceção da Crespa Repolhuda, tiveram uma germinação total alta (Figura 3), as alfaces Americana e Aurélia apresentaram germinação acima de 99\% em todos os tratamentos.

Figura 3. Germinação no teste de frio de cultivares de alface em função da aplicação de produtos biológicos estimulantes de desenvolvimento vegetal, UNESP Ilha Solteira (SP), 2021.

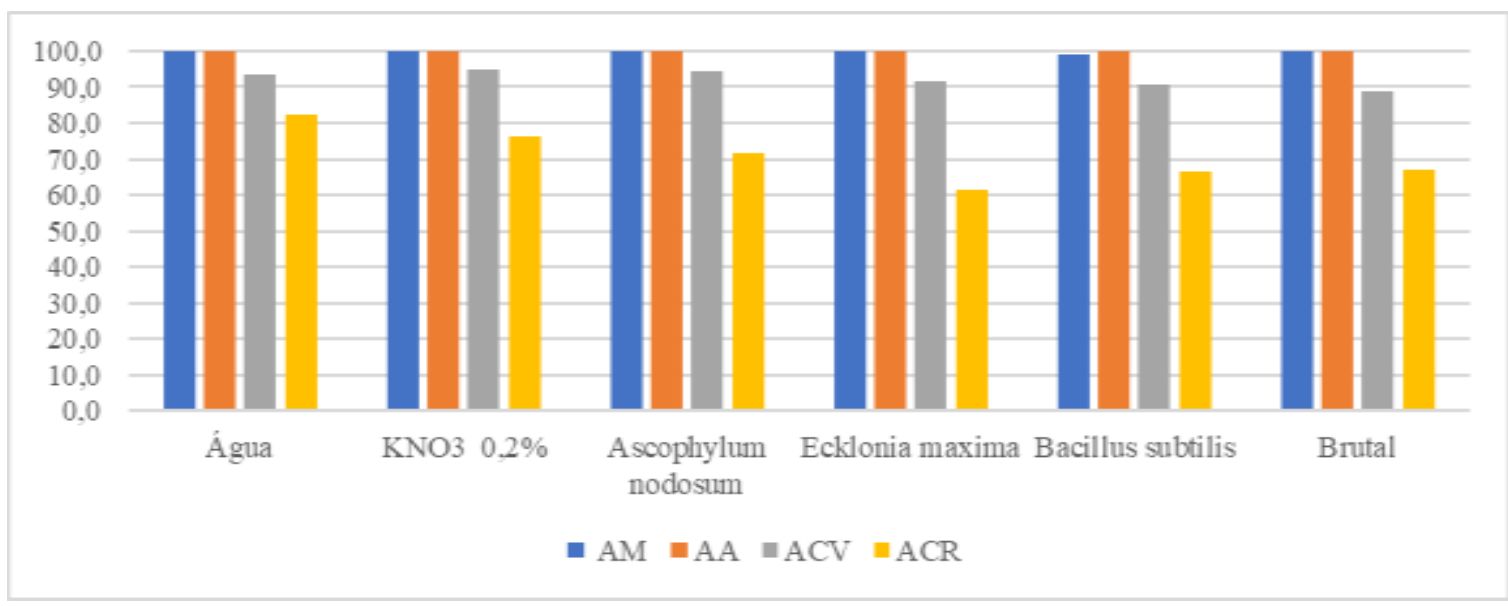

Fonte: Autores.

Na Tabela 5, assim como já esperado devido a sua baixa germinação, pode-se observar um menor índice de velocidade de germinação (IVG), para a alface Crespa Repolhuda. As alfaces Aurélia, Americana e a Crespa de Verão não diferiram entre os tratamentos. Maior rapidez de germinação na primeira contagem evidencia uma maior uniformidade de desenvolvimento, o que é fundamental para colheita e comercialização do produto. 
Tabela 5. Índice de Velocidade de Germinação no teste de frio de cultivares de alface em função da aplicação de produtos biológicos estimulantes de desenvolvimento vegetal, UNESP Ilha Solteira (SP), 2021.

\begin{tabular}{|c|c|c|c|c|c|c|}
\hline \multirow{2}{*}{ Cultivares } & \multicolumn{6}{|c|}{ Tratamentos } \\
\hline & T1 & $\mathbf{T} 2$ & T3 & T4 & T5 & T6 \\
\hline AA & $16,64 \mathrm{Aa}$ & $16,58 \mathrm{Aa}$ & $16,64 \mathrm{Aa}$ & $16,64 \mathrm{Aa}$ & $16,64 \mathrm{Aa}$ & $16,54 \mathrm{Aa}$ \\
\hline ACR & $12,11 \mathrm{Ab}$ & $11,17 \mathrm{ABb}$ & $10,46 \mathrm{BCb}$ & $8,65 \mathrm{Dc}$ & 9,73 BCDc & 9,38 CDc \\
\hline $\mathrm{ACV}$ & $15,39 \mathrm{Aa}$ & $15,60 \mathrm{Aa}$ & $15,45 \mathrm{Aa}$ & $14,86 \mathrm{Ab}$ & $14,80 \mathrm{Ab}$ & $14,76 \mathrm{Ab}$ \\
\hline $\mathrm{AM}$ & $16,67 \mathrm{Aa}$ & $16,62 \mathrm{Aa}$ & $16,61 \mathrm{Aa}$ & $16,67 \mathrm{Aa}$ & $16,47 \mathrm{Aa}$ & $16,67 \mathrm{Aa}$ \\
\hline \multirow{3}{*}{$\mathrm{F}$} & \multicolumn{3}{|c|}{ Cultivares } & \multicolumn{3}{|c|}{$382,99^{* *}$} \\
\hline & \multicolumn{3}{|c|}{ Tratamentos $(\mathrm{T})$} & \multicolumn{3}{|c|}{$4,46^{* *}$} \\
\hline & \multicolumn{3}{|c|}{ Cultivares x $\mathrm{T}$} & \multicolumn{3}{|c|}{$2,58^{* *}$} \\
\hline \multirow{2}{*}{ DMS } & \multicolumn{3}{|c|}{ Cultivares } & \multicolumn{3}{|c|}{0,57} \\
\hline & \multicolumn{3}{|c|}{ Tratamentos } & \multicolumn{3}{|c|}{1,57} \\
\hline C.V. $(\%)$ & & & & \multicolumn{3}{|c|}{5,16} \\
\hline
\end{tabular}

Médias seguidas de letras diferentes maiúsculas nas linhas e minúsculas nas colunas, diferem estatisticamente entre si, Teste de Tukey, $\mathrm{p}<0.05$ ) (means followed by different uppercase letters in the lines and lowercase letters in the columns, differ statistically from each Other, Tukey, p<0.05). Cultivares: ACR-Crespa Repolhuda, ACV-Crespa de Verão, AM-Americana e AA-Aurélia; Tratamentos: T1-Água (testemunha), T2-Nitrato de Potássio ( $\mathrm{KNO}_{3}$ 0,2\%), T3-Ascophylum nodosum, T4-Ecklonia maxima, T5Brutal ${ }^{\circledR}$ (Complexo de micro-organismos) e T6-Serenade ${ }^{\circledR}$ (Bacillus subtilis). Fonte: Autores.

Na Figura 4, observa-se que o uso de Ecklonia maxima e o produto Brutal@ na cultivar Americana apresentou maior massa seca comparado com os demais, o mesmo aconteceu com a cultivar Crespa Repolhuda; já o uso de Bacillus subtilis foi bom nas cultivares Aurélia e Crespa Repolhuda.

Figura 4. Matéria Seca (g) no teste de frio de cultivares de alface em função da aplicação de produtos biológicos estimulantes de desenvolvimento vegetal, UNESP Ilha Solteira (SP), 2021.

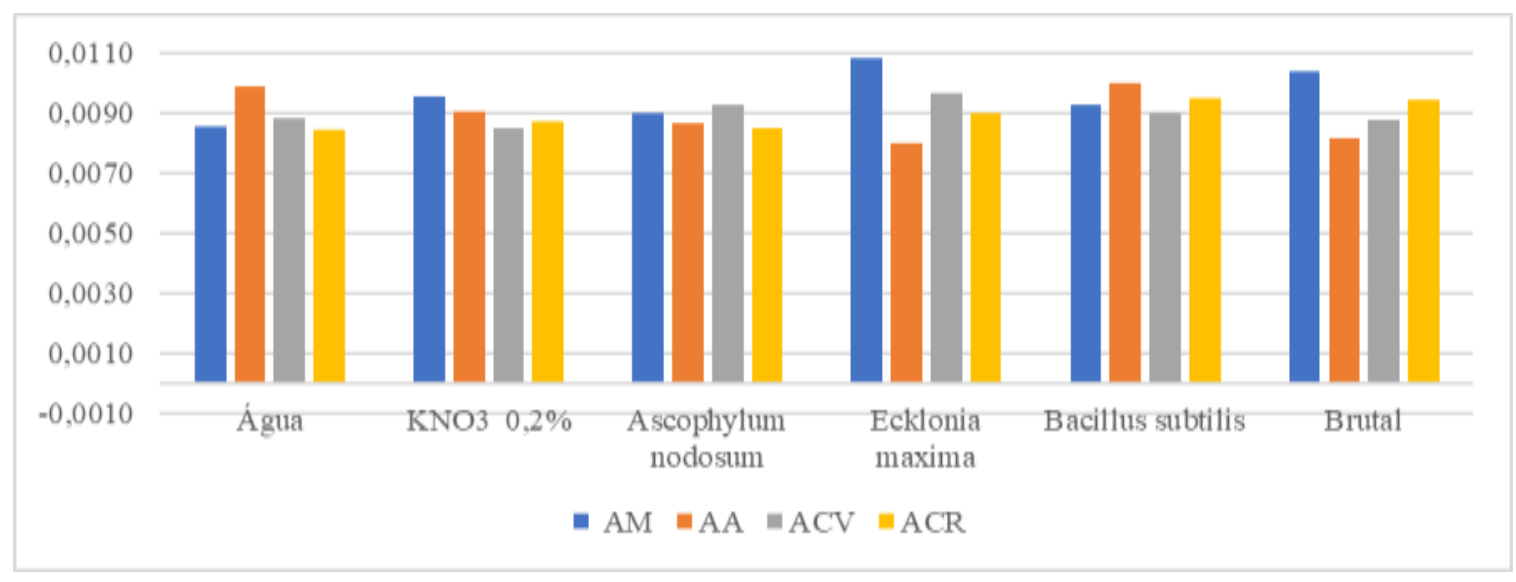

Fonte: Autores.

Com o teste de Envelhecimento acelerado, ao submeter as sementes à alta temperatura e alta umidade, o lote de Alface Aurélia demonstrou alto vigor como pode ser observado na Figura 5, sua germinação final se manteve acima de $97 \%$. Para todos os tratamentos, as sementes da cultivar de alface Americana apresentaram um desempenho variável com valores na faixa de $70 \%$ nos tratamentos com $\mathrm{KNO}_{3} 0,2 \%$ e Ecklonia maxima e inferior nos demais. Já as sementes dos cultivares Alface 
Crespa de Verão e Alface Crespa Repolhuda evidenciaram um desempenho inadequado, indicando que as sementes se mostraram sensíveis à aplicação da alta temperatura.

Figura 5. Germinação no teste de envelhecimento de cultivares de alface em função da aplicação de produtos biológicos estimulantes de desenvolvimento vegetal, UNESP Ilha Solteira (SP), 2021.

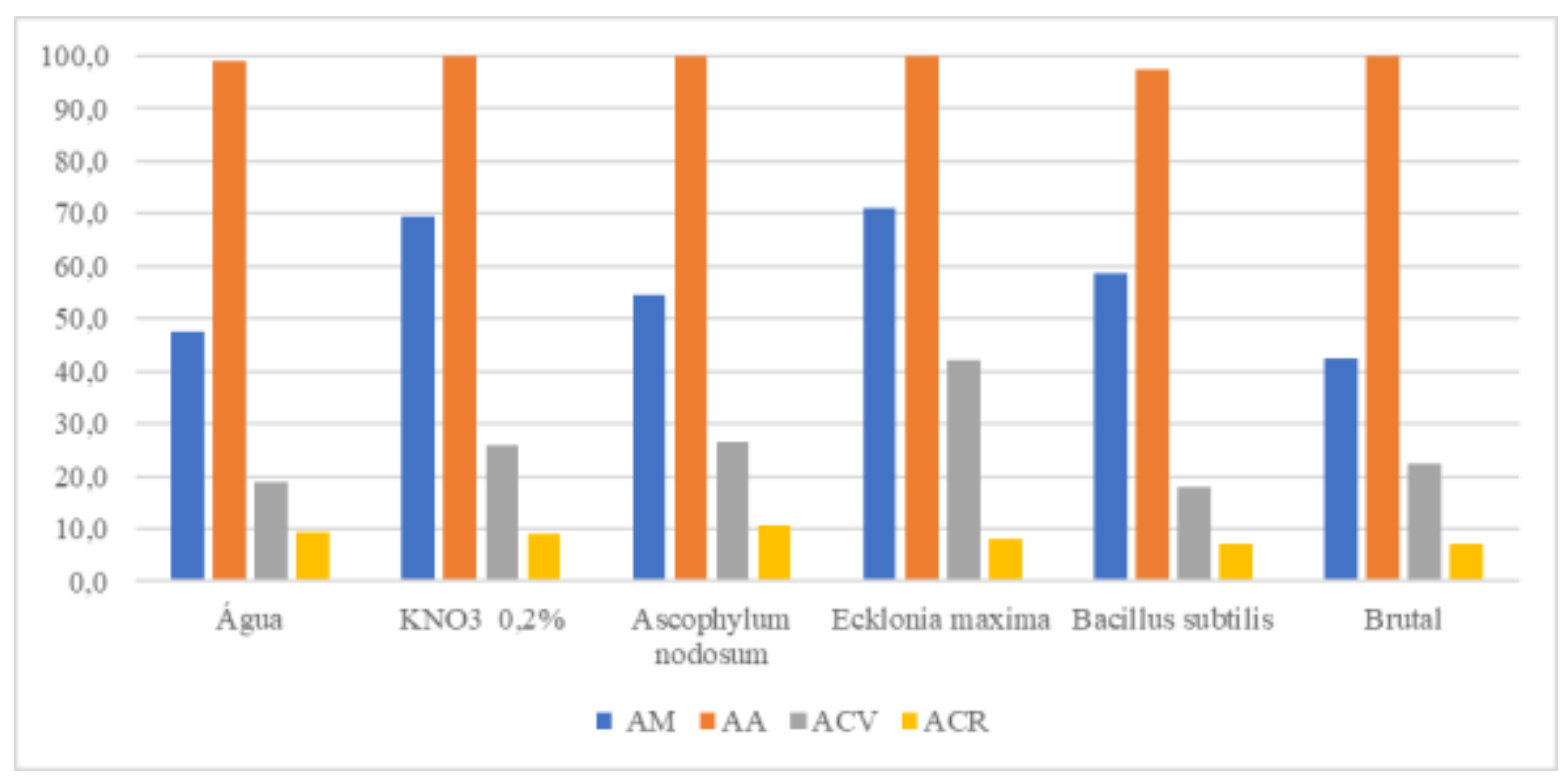

Fonte: Autores.

Estes resultados são importante indicativos do comportamento germinativo das sementes após serem expostas às condições de alta temperatura, fato que permite fazer a indicação de determinado cultivar para uma região, informações estas que associadas aos resultados do teste de frio permite este tipo de direcionamento (Marcos Filho, 2021).

Observa-se que a ação dos produtos não evidenciou um incremento ao desempenho das sementes, e para esta situação, os dados não evidenciaram o mesmo comportamento verificado para a primeira contagem e velocidade de germinação. Como o teste de germinação é realizado em condições ótimas (temperatura, água e oxigênio) para cada espécie a ação dos produtos se mostraram mais evidentes e não limitantes ao desempenho das sementes. Já no envelhecimento, principalmente a alta temperatura pode ter restringido o desempenho das sementes e como muitos cultivares apresentam desempenho superior em condições mais amenas, o efeito da alta temperatura se mostrou restritivo ao desempenho fisiológico das sementes. De acordo com Gray (1975) citado por Bueno et al. (2008), sementes de alguns cultivares de alface podem germinar em temperaturas variando de 5 a $33^{\circ} \mathrm{C}$; entretanto de maneira geral, temperaturas acima de $30^{\circ} \mathrm{C}$ afetam a germinação, reduzindo a velocidade ou a porcentagem de germinação.

Assim, acredita-se que no presente trabalho a redução na germinação e no crescimento das plântulas das sementes expostas ao teste de envelhecimento é um indicativo que as sementes das três cultivares foram afetadas por esta condição desfavorável (41 $\left.{ }^{\circ} \mathrm{C} / 72 \mathrm{~h}\right)$. Conforme Sung (1996), o endosperma de sementes de alface atua como uma barreira física à emissão da radícula sob condições de altas temperaturas, uma vez que as paredes celulares do endosperma são compostas principalmente de polímeros de galactomananas e endo-B-manase, que poderia ser uma enzima potencialmente regulatória no enfraquecimento do endosperma.

Nascimento et al. (2012) relataram que a exposição das sementes de alface à temperatura elevada durante o processo de inibição, pode acarretar fenômenos como a termoinibição e a termodormência, correspondendo respectivamente à inibição temporária e completa da germinação em decorrência do enrijecimento do endosperma, o qual restringe a protusão da radícula. 
Avaliando a matéria seca das plantas (Tabela 6), o tratamento com Bacillus resultou em uma menor massa para as cultivares Crespa Repolhuda e Crespa de Verão, o que não se repetiu para as cultivares Aurélia e Americana. Não houve diferença estatística entre os tratamentos para as cultivares Aurélia e Americana.

Da mesma forma, a massa de matéria seca das plântulas no envelhecimento indicaram desempenho semelhante ao das plântulas normais e não confirmaram o verificado no teste de germinação, primeira contagem e teste de frio.

Tabela 6. Matéria Seca (g) no teste de envelhecimento de cultivares de alface em função da aplicação de produtos biológicos estimulantes de desenvolvimento vegetal, UNESP Ilha Solteira (SP), 2021.

\begin{tabular}{|c|c|c|c|c|c|c|}
\hline \multirow{2}{*}{ Cultivares } & \multicolumn{6}{|c|}{ Tratamentos } \\
\hline & $\mathbf{T 1}$ & $\mathbf{T} 2$ & T3 & $\mathbf{T 4}$ & T5 & T6 \\
\hline AA & $0,0112 \mathrm{Aa}$ & $0,0117 \mathrm{Aa}$ & 0,0110 Aa & $0,0113 \mathrm{Aa}$ & 0,0109 Aa & $0,0112 \mathrm{Aa}$ \\
\hline ACR & $0,0060 \mathrm{Ac}$ & $0,0058 \mathrm{Ab}$ & $0,0066 \mathrm{Ab}$ & $0,0047 \mathrm{Ab}$ & $0,0054 \mathrm{Ab}$ & $0,0053 \mathrm{Ab}$ \\
\hline $\mathrm{ACV}$ & 0,0068 BCbc & $0,0106 \mathrm{Aa}$ & $0,0070 \mathrm{BCb}$ & $0,0112 \mathrm{Aa}$ & $0,0053 \mathrm{Cb}$ & $0,0085 \mathrm{ABa}$ \\
\hline $\mathrm{AM}$ & 0,0094 Aab & $0,0103 \mathrm{Aa}$ & $0,0104 \mathrm{Aa}$ & $0,0092 \mathrm{Aa}$ & 0,0097 Aa & 0,0090 Aa \\
\hline \multirow{3}{*}{$\mathrm{F}$} & \multicolumn{3}{|c|}{ Cultivares } & \multicolumn{3}{|c|}{$63,71^{* *}$} \\
\hline & \multicolumn{3}{|c|}{ Tratamentos $(\mathrm{T})$} & \multicolumn{3}{|c|}{$2,93^{* *}$} \\
\hline & \multicolumn{3}{|c|}{ Cultivares x T } & \multicolumn{3}{|c|}{$2,98^{* *}$} \\
\hline \multirow{2}{*}{ DMS } & \multicolumn{3}{|c|}{ Cultivares } & \multicolumn{3}{|c|}{0,001} \\
\hline & \multicolumn{3}{|c|}{ Tratamentos } & \multicolumn{3}{|c|}{0,003} \\
\hline C.V. $(\%)$ & & & & \multicolumn{3}{|c|}{16,74} \\
\hline
\end{tabular}

Médias seguidas de letras diferentes maiúsculas nas linhas e minúsculas nas colunas, diferem estatisticamente entre si, Teste de Tukey, $\mathrm{p}<0.05$ ) (means followed by different uppercase letters in the lines and lowercase letters in the columns, differ statistically from each Other, Tukey, p<0.05). Cultivares: ACR-Crespa Repolhuda, ACV-Crespa de Verão, AM-Americana e AA-Aurélia; Tratamentos: T1-Água (testemunha), T2-Nitrato de Potássio ( $\mathrm{KNO}_{3}$ 0,2\%), T3-Ascophylum nodosum, T4Ecklonia maxima, T5-Brutal ${ }^{\circledR}$ (Complexo de micro-organismos) e T6-Serenade ${ }^{\circledR}$ (Bacillus subtilis). Fonte: Autores.

No entanto, como a massa de matéria seca na forma avaliada depende da taxa de germinação, os resultados obtidos refletem a menor taxa e também indicam que a alta temperatura restringiu no desenvolvimento das plantas.

\section{Conclusão}

Há comportamento diferente das cultivares em relação à aplicação dos produtos contendo rizobactérias, $\mathrm{KNO}_{3}$ ou complexo de micro-organismos.

O efeito dos produtos é mais pronunciado nas sementes de menor vigor.

O teste de envelhecimento na forma em que foi realizado não evidenciou o desempenho das sementes.

\section{Referências}

ABCSEM. (2013). Associação Brasileira do Comércio de Sementes e Mudas. O mercado de folhosas: números e tendências. http://www.abcsem.com.br/upload/arquivos/O_mercado_de_folhosas__Numeros_e_Tendencias_-_Steven.pdf.

Acadian Agritech. (2009). Ciência das Plantas. Site Institucional da Acadian Agritech. http://www.acadianagritech.ca/portuguese/PSansA.htm.

Albrecht, L. P., Oliveira JR, R. S., Braccini, A. L., Alonso, D. G., Barbosa, M. C., \& Albrecht, A. J. P. (2010). Componentes de produção e qualidade fisiológica e sanitária de sementes de soja RR em resposta a aplicação de glyphosate. In: I Simpósio Internacional sobre Glyphosate, 2007, Botucatu. Anais... Botucatu: UNESP, 2007.Alvares, C. A. Stape, J. L., Sentelhas, P. C., De Moraes, G., Leonardo, J. \& Sparovek, G. Köppen's climate classification map for Brazil. Meteorologische Zeitschrift, 22(6): 711-728. 
Alvares, C. A., Stape, J. L., Sentelhas, P. C., Gonçalves, J. L. M., \& Sparovek, G. (2013). Köppen's climate classification map for Brazil. Meteorologische Zeitschrift, 22(6), 711-728.

Araujo, F. F. (2008). Inoculação de sementes com Bacillus subtilis, formulado com farinha de ostra e desenvolvimento de milho, soja e algodão. Ciência e Agrotecnologia, 2, 456-462.

Brasil. (2009) Ministério da Agricultura, Pecuária e Abastecimento. Regras para Análise de Sementes. Ministério da Agricultura, Pecuária e Abastecimento. Secretaria de Defesa Agropecuária. Brasília, DF: Mapa/ACS. 398p.

Bueno, A. C. R., Villela, R. P., Guimarães, R. M., Souza, R. J., Carvalho, B. O., \& Costa, R. R. (2008). Efeito do etrel e ácido giberélico na germinação de sementes de alface (Lactiva sativa L.) cultivar Simpson. Horticultura Brasileira. 26, 1381-1385.

Castro, P. R. C., \& Vieira, E. L. (2001). Aplicações de reguladores vegetais na agricultura tropical. Guaíba: Agropecuária, 132p.

Costa, C. P. D. A., \& Sala, F. C. (2005) A evolução da alfacicultura brasileira. Horticultura Brasileira, 23.

Decoteau, D. R. et al. (1995). The lettuce growing handbook: botany, field produces, growing problems, and postharvest handling. Illinois: Oak Brook, 60 p. Ferreira, D. F. (2011). Sisvar: um sistema computacional de análise estatística. Ciência e Agrotecnologia, 35(6), 1039-1042. https://doi.org/10.1590/S141370542011000600001

Ferreira, N. C., Mazzuchelli, R. C. L., Mazzuchelli, E. H. L., \& Araújo, F. F. (2013). Crescimento de mudas de alface em substrato associado a doses crescentes de Bacillus subtilis. Colloquium Agrarie, 9, 36-42.

Fetter, P. R. (2018). Hidrolisados de resíduos de raízes e caules de tabaco para estimulação da germinação de sementes de arroz e milho. Dissertação (Pós graduação em tecnologia Ambiental) Universidade de Santa Cruz do Sul, Santa Cruz do Sul. http://hdl.handle.net/11624/2249.

Fonseca, A, S., Thomazini, A., Bertossi, A. P. A., \& Amaral, J. F. T. (2013). Análise de crescimento e absorção de fósforo em alface. Revista Nucleus, v.10, n.2. http://dx.doi.org/10.3738/1982.2278.895 10.3738/1982.2278.895.

Gomes, T. M., Modolo, V. A., Botrel, T. A. \& Oliveira, R. F. (2005). Aplicação de CO2 via água de irrigação na cultura da alface. Horticultura Brasileira, 23:316-319.

IBGE. (2017). Instituto Brasileiro de Geografia e Estatística. Censo Agropecuário. https://sidra.ibge.gov.br/.

Lanna Filho, R., Ferro, H. M., \& Pinho, R. S. C. (2010). Controle biológico mediado por Bacillus subtilis. Revista Trópica: Ciências Agrárias e Biológicas, chapadinha-MA, 4(2), 12.

Lucy, M., Reed, E., \& Glick, B. R. (2004). Applications of free living plant growt-promoting rhizobacteria. Antonie Van Heewenhock, 86(1), 1-25.

Maguire, J. D. (1962). Speed of germination aid in selection and evaluation for seedling and vigour. Crop Science, Madison, 2(2), $176-177$.

Marcos Filho, J. (2021). Teste de envelhecimento acelerado. In Kranowski, F. C., Vieira, R. D., França Neto, J. B., Nakagawa, J., Marcos Filho. Testes de vigor em sementes: conceitos e teste. Abrantes, Londrina - PR.

Marcos Filho, J. (2015). Fisiologia de sementes de plantas cultivadas. (2a ed.), ABRATES, 660p.

Mariano, R. L. R., Silveira, E. B., Assis, S. M. P., Gomes, A. M. A, Nascimento, A. R. P, \& Donato, V. M. T. (2004). Importância de bactérias promotoras de crescimento e de biocontrole de doenças de plantas para uma agricultura sustentável. Anais da Academia Pernambucana de Ciência Agronomica, 1(1), 89111.

Martins, M. B. G., \& Castro, P. R. C. (1999). Effects of gibberellin and ethephon on the anatomy of sugar cane plants. Pesquisa Agropecuária Brasileira, $34(10), 1855-1863$.

Meirelles, A. F. M., Baldotto, M. A., \& Baldotto, L. E. B. (2017). Produtividade da alface (Lactuca sativa L.) em resposta a aplicação de ácidos húmicos e bactérias diazotroficas em condições de campo. Revista Ceres, 64(5), 553-556.

Melo, I. S. de. (2018). Rizobactérias. http://www.agencia.cnptia.embrapa.br/gestor/agricultura_e_meio_ambiente/arvore/CONTAG01_53_2102007 92814.html.

Mota, W. F., Pereira, R. D., Santos, G. S., Vieira, J. C. B. (2012). Agronomic and economic viability of intercropping onion and lettuce. Horticultura Brasileira, 30(2), 349-354.

Nascimento, W. M., Groda, M. D., \& Lopes, A. C. A. (2012). Produção de sementes, qualidade fisiológica e identificação de genótipos de alface termotolerantes. Revista Brasileira de sementes, 34(3), 510-517.

Ono, E. O., Rodrigues, J. D., \& Santos, S. O. (1999). Efeito de fitorreguladores sobre o desenvolvimento de feijoeiro (Phaseolus vulgaris L.) cv Carioca. Revista Biociências, 5(1), 7-13.

Rodrigues, J. D. (2008). Biorreguladores, aminoácidos e extratos de algas: verdades e mitos. Jornal Informações Agronômicas, (122), 15-17.

Rodrigues I. N., Lopes, M. T. G., Lopes, R. Gama, A. S., \& Milagres, C., P. (2007). Avaliação de cultivares de alface crespa para a região de Manaus. In: Congresso brasileiro de olericultura, 47. Resumos... Porto Seguro: ABH (CD-ROM). Santos, C. L., Seabra, Jr. S., Gadum de Lalla. J., Theodoro, V. C. A., Nespoli, A. 2009. Desempenho de cultivares de alface tipo crespa sob altas temperaturas em Cáceres-MT. Revista Agrarian 23: 87-98.

Viggiano J. (1990). Produção de sementes de alface. In: Castellane, P. D. (Eds). Produção de sementes de Hortaliças. Jaboticabal: FCAV/FUNEP. 
Research, Society and Development, v. 10, n. 12, e182101220353, 2021

(CC BY 4.0) | ISSN 2525-3409 | DOI: http://dx.doi.org/10.33448/rsd-v10i12.20353

Silva, C., Arrais, I. G., Almeida, J. P. N., Dantas, L. L. G, \& Mendonça, V. (2016) Extrato de algas Ascophylum nodosum (L.) na produção de porta enxerto de anonna glabla L. Revista de Ciência Agrarias, 39(2), 234-241.

Stirk, W. A., Novak, O., Strnad, M., \& Stadens, V. (2003). Extorinins in macro alga. Plant Growth Regulation, 41(1),13-24.

Sung, Y. (2008). Identification and caracterization of thermotolerance in lettuce seed germination. Gaines Ville, University of Flórida: 67, 1996. Horticultura Brasileira, 26(2).

Tekrony, D. M., \& Egli, D. S. (1995). Acumulation of seed vigour during development and maturation. Ellis, R. H., Black, M., Murdoch, A. J., \& Hong, T. D. (eds.), Basic and Applied Aspects of Seed Biology. Kluwer Academic Publishers, Dordrecht, 369-384.

Turini, T., Cahn, M., Cantwell, M., Jackson, L., Koike, S., Natwick, E., Smith, R., Subarrao, K., \& Takele, E. (2011). Iceberg lettuce production in California. http://anrcatalog.ucdavis.edu/ pdf/7215.pdf.

Vilela, N. J., \& Luengo, R. F. A. (2017). Produção de Hortaliças Folhosas no Brasil. Campo \& Negócios, Hortifruti, Uberlândia, ano XII, n. 146.

Zucareli, C., Barzan, R. R., Da Silva, J. B., \& Chaves, D. P. (2018). Associação de fosfatos e inoculação com Bacillus subtilis e seu efeito no crescimento e desempenho produtivo do feijoeiro. Revista Ceres, 65(2), 189-195. 